\title{
Searching for the definition of macrosomia through an outcome-based approach in low- and middle-income countries: a secondary analysis of the WHO Global Survey in Africa, Asia and Latin America
}

Jiangfeng Ye ${ }^{1,2}$, Maria Regina Torloni ${ }^{3}$, Erika Ota ${ }^{4}$, Kapila Jayaratne $^{5}$, Cynthia Pileggi-Castro $^{6}$, Eduardo Ortiz-Panozo $^{7}$, Pisake Lumbiganon ${ }^{8}$, Naho Morisaki ${ }^{9}$, Malinee Laopaiboon ${ }^{10}$, Rintaro Mori ${ }^{4}$, Özge Tunçalp ${ }^{2}$, Fang Fang ${ }^{1}$, Hongping $\mathrm{Yu}^{11}$, João Paulo Souza ${ }^{12}$, Joshua Peter Vogel ${ }^{2}$ and Jun Zhang ${ }^{1 *}$

\begin{abstract}
Background: No consensus definition of macrosomia currently exists among researchers and obstetricians. We aimed to identify a definition of macrosomia that is more predictive of maternal and perinatal mortality and morbidity in low- and middle-income countries.
\end{abstract}

Methods: We conducted a secondary data analysis using WHO Global Survey on Maternal and Perinatal Health data on Africa and Latin America from 2004 to 2005 and Asia from 2007 to 2008. We compared adverse outcomes, which were assessed by the composite maternal mortality and morbidity index (MMMI) and perinatal mortality and morbidity index (PMMI) in subgroups with birthweight (3000-3499 g [reference group], 3500-3999 g, 4000-4099 g, 4100-4199 g, 4200-4299 g, 4300-4399 g, 4400-4499 g, 4500-4999 g) or country-specific birthweight percentile for gestational age $\left(50^{\text {th }}-74^{\text {th }}\right.$ percentile [reference group], $75^{\text {th }}-89^{\text {th }}, 90^{\text {th }}-94^{\text {th }}, 95^{\text {th }}-96^{\text {th }}$, and $\geq 97^{\text {th }}$ percentile).

Two-level logistic regression models were used to estimate odds ratios of MMMI and PMMI.

Results: A total of 246,659 singleton term births from 363 facilities in 23 low- and middle-income countries were included. Adjusted odds ratios (aORs) for intrapartum caesarean sections exceeded 2.0 when birthweight was

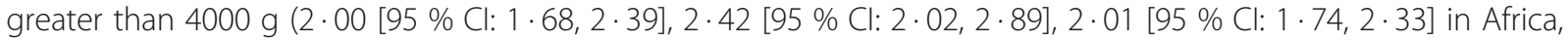
Asia and Latin America, respectively). aORs of MMMI reached 2.0 when birthweight was greater than $4000 \mathrm{~g}, 4500 \mathrm{~g}$ in Asia and Africa, respectively. aORs of PMMI approached to $2.0(1.78$ [95 \% Cl: 1. 16, 2.74]) when birthweight was greater than $4500 \mathrm{~g}$ in Latin America. When birthweight was at the $90^{\text {th }}$ percentile or higher, aORs of MMMl and PMMI increased, but none exceeded 2.0.

Conclusions: The population-specific definition of macrosomia using birthweight cut-off points irrespective of gestational age (4500 g in Africa and Latin America, $4000 \mathrm{~g}$ in Asia) is more predictive of maternal and perinatal adverse outcomes, and simpler to apply compared to the definition based on birthweight percentile for a given gestational age.

Keywords: Macrosomia, Maternal mortality, Maternal morbidity, Neonatal mortality, Neonatal morbidity

\footnotetext{
* Correspondence: junjimzhang@gmail.com

${ }^{1}$ Ministry of Education-Shanghai Key Laboratory of Children's Environmental Health, Xinhua Hospital, Shanghai Jiao Tong University School of Medicine,

Shanghai, China

Full list of author information is available at the end of the article
}

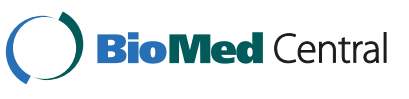

(c) 2015 Ye et al. Open Access This article is distributed under the terms of the Creative Commons Attribution 4.0 International License (http://creativecommons.org/licenses/by/4.0/), which permits unrestricted use, distribution, and reproduction in any medium, provided you give appropriate credit to the original author(s) and the source, provide a link to the Creative Commons license, and indicate if changes were made. The Creative Commons Public Domain Dedication waiver (http:// creativecommons.org/publicdomain/zero/1.0/) applies to the data made available in this article, unless otherwise stated. 


\section{Background}

"Macrosomia" is a term that describes a very large fetus or neonate. The condition may be caused by constitutional/ genetic factors, maternal obesity and/or excessive gestational weight gain, or maternal hyperglycemia due to preexisting diabetes or gestational diabetes that were not adequately controlled [1]. In low-and middle- income countries (LMICs) or settings where antenatal care is sub-optimal, poorly controlled diabetes or undiagnosed gestational diabetes may be a more important cause for macrosomia than in high-income countries, where antenatal care is better. In high-income countries, the prevalence of macrosomia has been increasing in the last two to three decades [2, 3]. But in many LMICs, macrosomia is still not perceived to have the same priority as other public health problems (e.g., HIV) [4]. However, with the increasing prevalence of maternal obesity and diabetes $[5,6]$ a parallel increase in macrosomic infants might be expected in LMICs. Complicated deliveries related to macrosomia could lead to more severe adverse outcomes in resource-poor settings due to limited availability of obstetric care. Thus, a precise definition of macrosomia that is more predictive of maternal and perinatal mortality and morbidity is needed. In this study, we aimed to explore a definition through an outcome-based approach and comparing commonly used definitions currently.

Currently, no consensus definition exists among researchers and obstetricians. The most commonly used definition is based on birthweight cut-off points (e.g., $4000 \mathrm{~g}$ or $4500 \mathrm{~g}$ ) [3, 7-9]. As it is increasingly recognized that racial variation in birthweight is substantial, more and more studies are using specific birthweight percentiles as cut-off points at a given gestational week (e.g., $\mathrm{P}_{90}$ or $\mathrm{P}_{97}$ ) based on the concept of large-for-gestational-age (LGA) [2, 10]. Furthermore, most studies on macrosomia have focused on Caucasian populations in high-income countries, and very few studies on the topic focus on LMICs [2].

In this study we analyzed data from 23 LMICs in Africa, Asia, and Latin America that participated in the World Health Organization (WHO) Global Survey on Maternal and Perinatal Health (2004-2008). We assessed commonly used definitions of the term 'macrosomia' through an outcome-based approach. Two types of definitions were compared: one based on empirical absolute birthweight and the other on the country-specific birthweight percentile at each gestational week. We aimed to identify a definition that was more predictive of maternal and perinatal mortality and morbidity in term pregnancies in LMICs, which also takes into account regional variation.

\section{Methods}

\section{Study design and data extraction}

The general objective of the WHO Global Survey on Maternal and Perinatal Health was to create a global database on health services and outcomes for maternal and perinatal health, which concentrated on the relationship between mode of delivery and perinatal outcomes $[2,11]$. This survey has previously been described in detail elsewhere $[2,11,12]$. A total of 373 facilities in 24 countries in Africa, Asia and Latin America participated in this survey. Data collection was carried out in 2004-05 in Africa and Latin America, and in 2007-08 in Asia. Trained data collectors extracted data from medical records and completed standardized forms. Gestational age was calculated based on the difference between the estimated and actual delivery date in the medical records. Data related to outcomes were obtained until discharge from the hospital. Maternal weight was defined differently as described previously: in Africa and Latin America, maternal weight was the weight recorded at the first antenatal care visit, while in Asia it was the weight at the last visit before delivery $[2,11]$.

This is a secondary data analysis using data from the WHO Global Survey on Maternal and Perinatal Health. The protocol of this survey was approved by the ethics committees at the $\mathrm{WHO}$ and in all participating centres [11]. We obtained permission to use this data from Department of Reproductive Health and Research, WHO. An individual informed consent was not obtained because in this survey data were extracted from medical records without individual identification [11].

For the purpose of this analysis, the study sample was restricted to: 1) LMICs; 2) singleton pregnancies; 3) live births or fresh stillbirth; 4) birthweight $\geq 1000 \mathrm{~g}$; and 5) term births (gestational age 37-42 weeks). As the analysis focused on LMICs, Japan was excluded. Macerated stillbirths were also excluded, as we were interested in mortality associated with delivery, but not pre-delivery mortality. Infants who had missing information on birthweight or gestational age were also excluded. The sample selection process is shown in Fig. 1.

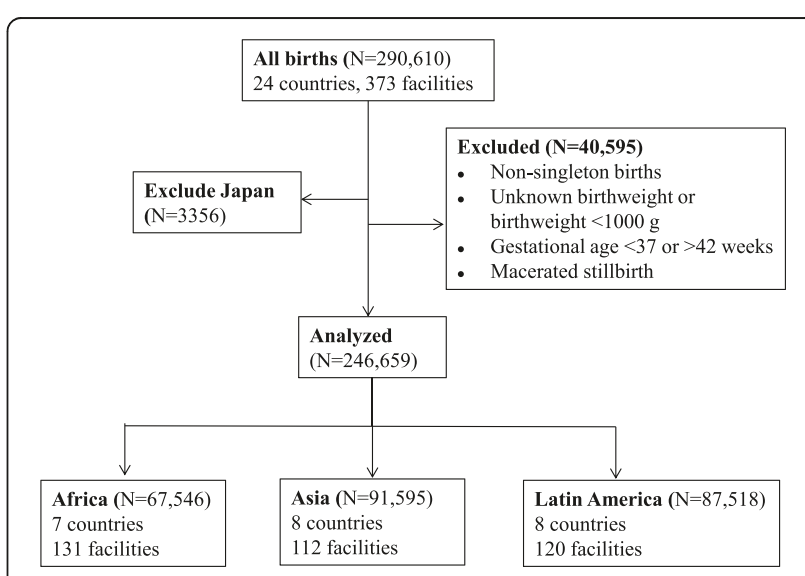

Fig. 1 Flow chart of inclusion and exclusion of study subjects 


\section{Statistical analysis}

Previous studies have shown that the perinatal mortality rate decreases with increasing birthweight or birthweight percentile until it reaches an inflection point, after which a further rise in birthweight increases the mortality, i.e., a reversed J-shaped mortality curve [13-17]. We applied this principle to our analysis: macrosomia was defined as a birthweight or birthweight percentile that exceeds the nadir of the mortality curve, and at which point the relative risk of perinatal mortality is twice or greater than that of the nadir. We used a priori odds ratio of $2 \cdot 0$ as a criterion for clinical significance, as per previous studies $[18,19]$.

We used the birthweight range of 3000-3499 g or at $50^{\text {th }}-74^{\text {th }}$ percentiles as the reference groups because our exploratory analyses showed that the nadir of the birthweight-specific mortality rate fell into these ranges. According to the exploratory analyses, there was no significant difference of risks of neonatal mortality and morbidity among subgroups of every $100 \mathrm{~g}$ between 3500 and $4000 \mathrm{~g}$. Thus, infants with birthweights in the range of 3500-3999 g were combined as one subgroup. Birthweights between 4000 and 4500 g were categorized into subgroups of every $100 \mathrm{~g}$ in order to explore the cut-off point for the definition of macrosomia.

To calculate weight percentiles, we adopted the global reference for fetal and birth weight percentile [20]. Briefly, based on the mean birthweight and variation at 40 weeks of gestation at each country, country-specific, equation-derived (i.e., not empirical) birthweight reference percentiles at $75^{\text {th }}, 90^{\text {th }}, 95^{\text {th }}$ and $97^{\text {th }}$ for each gestational week were generated. Infant birthweight was categorized according to these references.

The adverse maternal outcomes included maternal mortality and severe morbidity defined as any of the following: admission to an intensive-care unit (ICU), referral to a higher level or special care unit, blood transfusion, hysterectomy, vesico-vaginal/recto-vaginal fistula and third or fourth degree perineal laceration. The adverse perinatal outcomes were still-birth, early neonatal death (neonatal death within 7 days of birth) and severe neonatal morbidity defined as any of the following: admission to an Neonatal ICU for 7 days or more, referral to a higher level or special care unit and 5-min Apgar score less than four. As maternal and perinatal mortality and severe morbidity are rare outcomes, the composite maternal mortality and morbidity index (MMMI) and perinatal mortality and morbidity index (PMMI) were computed [2]. The MMMI and PMMI were coded as an event if mortality or any of the corresponding severe morbidity occurred. We also compared the risks of caesarean section and assisted vaginal delivery (forceps/vacuum extraction) for subgroups of infants by birthweight and birthweight percentile.
Two-level logistic regression models were used to estimate odds ratios (ORs) of maternal and perinatal mortality and morbidity by absolute birthweight and birthweight percentile for gestational age. Facilities represented units at level two and individuals within facilities were observations at level one. To take into account the large variations of anthropometry among regions, we stratified the analysis by region (Africa, Asia and Latin America). We adjusted for country, maternal age, marital status, education (total years of school attendance), obesity (maternal body mass index, BMI $\geq 30 \mathrm{~kg} / \mathrm{m}^{2}$ ), pre-existing diabetes, parity, infant sex, and gestational age as potential confounders according to previous literature [7, 10]. All analyses were conducted with SAS version 9.2 (SAS Institute Inc, Cary, NC).

Maternal weight and/or height, and consequently maternal BMI, were missing in more than $10 \%$ of the study population in Kenya (85 \%), Brazil (67\%), Angola (43\%), Argentina (33 \%), Uganda (21 \%) and Peru (13\%). A missing category within "maternal obesity" $\left(\mathrm{BMI} \geq 30 \mathrm{~kg} / \mathrm{m}^{2}\right)$ was therefore created and included in all regression models. In our sensitivity analysis, we restricted our calculations to countries with less than $10 \%$ of the missing value for BMI. The "(pre-existing) diabetes mellitus" variable was missing in less than $1 \%$ of the study population of each region. For the estimation of risks of MMMI and PMMI by birthweight or birthweight percentile, we performed a sensitivity analysis by excluding the variables "maternal obesity" and "diabetes" from the regression models. In another sensitivity analysis, we compared the adjusted odds ratios (aORs) of MMMI and PMMI in subgroups using birthweight percentile of the study population as cut-off points, the results were essentially unchanged (data not shown). In another sensitivity analysis, we compared the aORs of MMMI and PMMI in subgroups using the empirical country- and gestational-age-specific birthweight percentile of the study population as cut-off points, the results were essentially unchanged (not shown).

\section{Results}

A total of 246,659 deliveries at 363 facilities in 23 LMICs were included in this analysis (Fig. 1). Large variations in birthweight distribution were observed among infants in the three regions. Mean birthweight was $3037 \mathrm{~g}, 3225 \mathrm{~g}$ and $3253 \mathrm{~g}$ in Asia, Africa and Latin America, respectively, and the proportion of infants with a birthweight greater than $4500 \mathrm{~g}$ was $0.3 \%, 1 \cdot 2 \%$, and $0.7 \%$ across these three regions. The prevalence of birthweight greater than the $97^{\text {th }}$ percentile was $7.2 \%$ (Latin America), $10.1 \%$ (Africa) and $10.5 \%$ (Asia) in the three regions (Table 1). In all three regions, the proportion of maternal age older than 35 years, maternal obesity $\left(B M I \geq 30 \mathrm{~kg} / \mathrm{m}^{2}\right)$, maternal preexisting gestational diabetes, multiparas and infant 
Table 1 Country-specific birthweight distribution of singleton term births

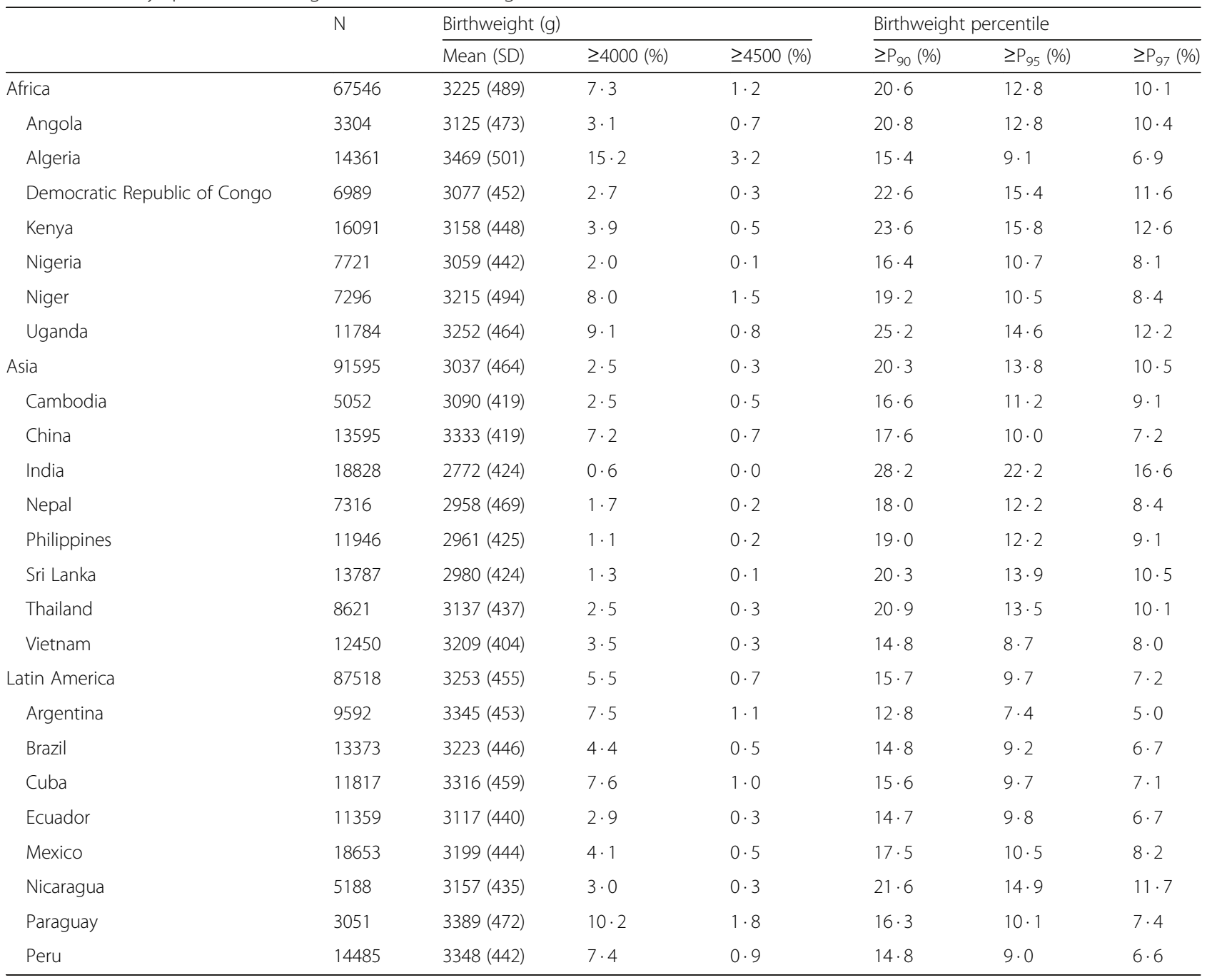

male sex was positively associated with higher birthweight (all $p$ values $<0 \cdot 0001$, Additional file 1: Table S1).

The overall rates of caesarean deliveries (and intrapartum rate) in Africa, Asia and Latin America were $12.0 \%(6 \cdot 2 \%), 28 \cdot 0 \%(12.5 \%)$ and $34.1 \%(12.0 \%)$ $(\mathrm{p}<0 \cdot 0001)$, respectively. Table 2 shows that compared with the reference group (3000-3499 g), aORs of elective and intrapartum caesarean section exceeded $2 \cdot 0$ when birthweight was greater than $4000 \mathrm{~g}$ in all three regions, while the risks of forceps or vacuum extraction did not rise significantly in most subgroups of infants in Asia and Latin America. In Africa, the aORs of forceps or vacuum extraction reached 2.0 when birthweight exceeded $4500 \mathrm{~g}$. There was a large variation of elective caesarean section rate among populations. In Africa, only around $5 \%$ suspected macrosomic babies were born through elective caesarean section while in Asia and Latin America, the responding rate was as high as around 40 and $20 \%$, respectively. The association between caesarean section and birthweight seemed less pronounced when infants were categorized by birthweight percentile and aORs hardly reached 2.0 (Table 3). The most important indication for CS for suspected macrosomic cases in all populations were cephalopelvic disproportion, followed by previous caesarean and fetal distress. Around $40 \%$ of suspected macrosomic cases born through caesarean had an indication of previous caesarean section. The other two indications accounted for around 30 and $20 \%$ caesarean deliveries, respectively (Additional file 2: Table S2).

The birthweight-specific risks of MMMI and PMMI are presented in Fig. 2. The association is expressed in a reverse "J"- or "U"-shaped curves. In all three regions, the lowest risk of MMMI corresponded to the birthweight range of 3000-3500 g, which was used as the reference group. When the birthweight exceeded $3500 \mathrm{~g}$, aORs of MMMI increased gradually. The aORs of MMMI reached 2. 0 when birthweight was greater than $4000 \mathrm{~g}, 4500 \mathrm{~g}$ and $5000 \mathrm{~g}$ in Asia, Africa and Latin 
Table 2 Prevalence and odds ratios of elective caesarean, intrapartum caesarean section and forceps or vacuum extraction by birthweight

\begin{tabular}{|c|c|c|c|c|c|c|c|c|c|c|}
\hline \multirow{2}{*}{$\begin{array}{l}\text { Birthweight } \\
\text { (g) }\end{array}$} & \multirow[b]{2}{*}{$\mathrm{N}$} & \multicolumn{3}{|c|}{ Elective caesarean section } & \multicolumn{3}{|c|}{ Intrapartum caesarean section } & \multicolumn{3}{|c|}{ Forceps extraction or vacuum extraction } \\
\hline & & Prevalence (\%) & Adjusted OR $(95 \% \mathrm{Cl})^{\mathrm{a}}$ & $p$ value & Prevalence (\%) & Adjusted OR $(95 \% \mathrm{Cl})^{\mathrm{a}}$ & $p$ value & Prevalence (\%) & Adjusted OR $(95 \% \mathrm{Cl})^{\mathrm{a}}$ & $p$ value \\
\hline Africa & 67546 & & & & & & & & & \\
\hline 3000-3499 & 28788 & 1.9 & $1 \cdot 00$ & - & $5 \cdot 8$ & $1 \cdot 00$ & - & $1 \cdot 8$ & $1 \cdot 00$ & - \\
\hline 3500-3999 & 15824 & 2.3 & $1.19(1.03,1.39)$ & 0.0196 & $6 \cdot 4$ & $1 \cdot 27(1 \cdot 16,1 \cdot 38)$ & $<0 \cdot 0001$ & $2 \cdot 4$ & $1 \cdot 22(1 \cdot 05,1 \cdot 41)$ & 0.0094 \\
\hline 4000-4099 & 2154 & 2.1 & $1.14(0.79,1.63)$ & 0.4824 & $9 \cdot 0$ & $2 \cdot 00(1 \cdot 68,2 \cdot 39)$ & $<0 \cdot 0001$ & $2 \cdot 9$ & $1 \cdot 81(1 \cdot 35,2 \cdot 44)$ & 0.0001 \\
\hline $4100-4199$ & 670 & 4.2 & $1.96(1.25,3.07)$ & 0.0032 & $8 \cdot 1$ & $1 \cdot 87(1 \cdot 38,2 \cdot 53)$ & $0 \cdot 0001$ & $3 \cdot 4$ & $1 \cdot 64(1 \cdot 03,2 \cdot 6)$ & $0 \cdot 0358$ \\
\hline $4200-4299$ & 686 & 5.0 & $2.61(1.72,3.96)$ & $<0 \cdot 0001$ & $9 \cdot 6$ & $2 \cdot 36(1 \cdot 78,3 \cdot 12)$ & $<0 \cdot 0001$ & $2 \cdot 2$ & $1 \cdot 09(0 \cdot 63,1 \cdot 88)$ & $0 \cdot 7618$ \\
\hline 4300-4399 & 377 & 2.0 & $0.83(0.36,1.93)$ & 0.6687 & $10 \cdot 6$ & $2 \cdot 39(1 \cdot 67,3 \cdot 42)$ & $<0 \cdot 0001$ & $2 \cdot 7$ & $0 \cdot 98(0 \cdot 50,1 \cdot 92)$ & $0 \cdot 9555$ \\
\hline 4400-4499 & 239 & 5.7 & $3.26(1.67,6.34)$ & 0.0005 & $8 \cdot 8$ & $2 \cdot 48(1 \cdot 52,4 \cdot 04)$ & $0 \cdot 0003$ & $3 \cdot 3$ & $1 \cdot 84(0 \cdot 86,3 \cdot 93)$ & $0 \cdot 1131$ \\
\hline 4500-4999 & 648 & 6.3 & $3.62(2.40,5.46)$ & $<0 \cdot 0001$ & $13 \cdot 0$ & $3 \cdot 78(2 \cdot 90,4 \cdot 92)$ & $<0 \cdot 0001$ & $3 \cdot 7$ & $2 \cdot 17(1 \cdot 37,3 \cdot 42)$ & $0 \cdot 0009$ \\
\hline$\geq 5000$ & 146 & 5.0 & $2.99(1.12,7.95)$ & 0.0287 & $14 \cdot 4$ & $5 \cdot 11(3 \cdot 00,8 \cdot 72)$ & $<0.0001$ & $8 \cdot 9$ & $8 \cdot 34(4 \cdot 22,16 \cdot 5)$ & $<0.0001$ \\
\hline Asia & 91595 & & & & & & & & & \\
\hline 3000-3499 & 36479 & 11.8 & $1 \cdot 00$ & - & $13 \cdot 2$ & $1 \cdot 00$ & - & $2 \cdot 7$ & $1 \cdot 00$ & - \\
\hline 3500-3999 & 13539 & 18.3 & $1.36(1.27,1.47)$ & $<0.0001$ & $18 \cdot 6$ & $1 \cdot 44(1 \cdot 36,1 \cdot 53)$ & $<0 \cdot 0001$ & $2 \cdot 6$ & $1 \cdot 27(1 \cdot 12,1 \cdot 45)$ & 0.0003 \\
\hline 4000-4099 & 945 & 31.6 & $2.33(1.90,2.86)$ & $<0 \cdot 0001$ & $24 \cdot 1$ & $2 \cdot 42(2 \cdot 02,2 \cdot 89)$ & $<0 \cdot 0001$ & $2 \cdot 1$ & $1 \cdot 64(1 \cdot 02,2 \cdot 64)$ & $0 \cdot 0413$ \\
\hline 4100-4199 & 492 & 34.8 & $2.32(1.76,3.07)$ & $<0 \cdot 0001$ & $26 \cdot 6$ & $2 \cdot 61(2 \cdot 05,3 \cdot 33)$ & $<0 \cdot 0001$ & $2 \cdot 2$ & $1 \cdot 69(0 \cdot 87,3 \cdot 29)$ & $0 \cdot 1195$ \\
\hline 4200-4299 & 320 & 40.3 & $3.19(2.28,4.47)$ & $<0 \cdot 0001$ & $28 \cdot 1$ & $3 \cdot 79(2 \cdot 77,5 \cdot 18)$ & $<0 \cdot 0001$ & $3 \cdot 1$ & $2 \cdot 73(1 \cdot 37,5 \cdot 46)$ & $0 \cdot 0045$ \\
\hline 4300-4399 & 183 & 40.5 & $2.63(1.70,4.07)$ & $<0 \cdot 0001$ & $30 \cdot 1$ & $3 \cdot 29(2 \cdot 22,4 \cdot 88)$ & $<0 \cdot 0001$ & $2 \cdot 2$ & $2 \cdot 56(0 \cdot 87,7 \cdot 55)$ & $0 \cdot 0884$ \\
\hline $4400-4499$ & 101 & 47.6 & $3.45(1.90,6.25)$ & $<0 \cdot 0001$ & $29 \cdot 7$ & $4 \cdot 13(2 \cdot 38,7 \cdot 15)$ & $<0 \cdot 0001$ & $2 \cdot 0$ & $1 \cdot 92(0 \cdot 42,8 \cdot 67)$ & $0 \cdot 3969$ \\
\hline 4500-4999 & 200 & 50.0 & $5.48(3.45,8.70)$ & $<0 \cdot 0001$ & $30 \cdot 0$ & $5 \cdot 20(3 \cdot 45,7 \cdot 83)$ & $<0 \cdot 0001$ & $2 \cdot 0$ & $2 \cdot 47(0 \cdot 81,7 \cdot 57)$ & $0 \cdot 1124$ \\
\hline$\geq 5000$ & 52 & 40.0 & $4.20(1.71,10.34)$ & 0.0018 & $32 \cdot 7$ & $9 \cdot 44(3 \cdot 99,22 \cdot 35)$ & $<0 \cdot 0001$ & $1 \cdot 9$ & $2 \cdot 38(0 \cdot 3,18 \cdot 81)$ & $0 \cdot 4113$ \\
\hline Latin America & 87518 & & & & & & & & & \\
\hline 3000-3499 & 38930 & 15.3 & $1 \cdot 00$ & - & $11 \cdot 2$ & $1 \cdot 00$ & - & $1 \cdot 2$ & $1 \cdot 00$ & - \\
\hline 3500-3999 & 20875 & 16.9 & $1.19(1.13,1.26)$ & $<0 \cdot 0001$ & $14 \cdot 0$ & $1 \cdot 28(1 \cdot 21,1 \cdot 35)$ & $<0 \cdot 0001$ & $1 \cdot 5$ & $1 \cdot 51(1 \cdot 30,1 \cdot 77)$ & $<0 \cdot 0001$ \\
\hline 4000-4099 & 1675 & 21.7 & $1.64(1.40,1.91)$ & $<0 \cdot 0001$ & $18 \cdot 1$ & $2 \cdot 01(1 \cdot 74,2 \cdot 33)$ & $<0 \cdot 0001$ & $0 \cdot 7$ & $0 \cdot 80(0 \cdot 43,1 \cdot 48)$ & 0.4699 \\
\hline $4100-4199$ & 1020 & 23.0 & $1.92(1.58,2.34)$ & $<0 \cdot 0001$ & $19 \cdot 1$ & $2 \cdot 19(1 \cdot 83,2 \cdot 62)$ & $<0 \cdot 0001$ & $1 \cdot 3$ & $1 \cdot 45(0 \cdot 79,2 \cdot 65)$ & $0 \cdot 2318$ \\
\hline $4200-4299$ & 757 & 25.1 & $2.01(1.60,2.51)$ & $<0 \cdot 0001$ & $21 \cdot 7$ & $2 \cdot 48(2 \cdot 03,3 \cdot 03)$ & $<0 \cdot 0001$ & $1 \cdot 5$ & $1 \cdot 88(0 \cdot 99,3 \cdot 58)$ & 0.0551 \\
\hline $4300-4399$ & 466 & 22.6 & $1.95(1.45,2.61)$ & $<0 \cdot 0001$ & $24 \cdot 4$ & $3 \cdot 00(2 \cdot 35,3 \cdot 84)$ & $<0 \cdot 0001$ & $0 \cdot 9$ & $0 \cdot 90(0 \cdot 32,2 \cdot 50)$ & $0 \cdot 8354$ \\
\hline $4400-4499$ & 281 & 28.7 & $2.59(1.83,3.66)$ & $<0 \cdot 0001$ & $19 \cdot 6$ & $2 \cdot 66(1 \cdot 89,3 \cdot 73)$ & $<0 \cdot 0001$ & $1 \cdot 1$ & $1 \cdot 81(0 \cdot 55,5 \cdot 94)$ & $0 \cdot 3257$ \\
\hline 4500-4999 & 569 & 38.1 & $3.66(2.87,4.68)$ & $<0 \cdot 0001$ & $23 \cdot 8$ & $4 \cdot 17(3 \cdot 29,5 \cdot 3)$ & $<0 \cdot 0001$ & $1 \cdot 1$ & $1 \cdot 98(0 \cdot 84,4 \cdot 63)$ & $0 \cdot 1163$ \\
\hline$\geq 5000$ & 55 & 35.3 & $3.54(1.67,7.53)$ & 0.0010 & $20 \cdot 0$ & $3 \cdot 13(1 \cdot 43,6 \cdot 85)$ & $0 \cdot 0045$ & $3 \cdot 6$ & $4 \cdot 64(0 \cdot 95,22 \cdot 64)$ & 0.0579 \\
\hline
\end{tabular}

a All estimates were based on two-level logistic regression mate observations at level one. We adjusted for country, maternal age, marital status, education (total years of school attendance), obesity, diabetes, parity, infant sex, and gestational age 
Table 3 Prevalence and odds ratios of elective caesarean, intrapartum caesarean section and forceps or vacuum extraction by birthweight percentile

\begin{tabular}{|c|c|c|c|c|c|c|c|c|c|c|}
\hline \multirow{2}{*}{$\begin{array}{l}\text { Birthweight } \\
\text { percentile }\end{array}$} & \multirow[b]{2}{*}{$\mathrm{N}$} & \multicolumn{3}{|c|}{ Elective caesarean section } & \multicolumn{3}{|c|}{ Intrapartum caesarean section } & \multicolumn{3}{|c|}{ Forceps extraction or vacuum extraction } \\
\hline & & $\begin{array}{l}\text { Prevalence } \\
(\%)\end{array}$ & $\begin{array}{l}\text { Adjusted OR } \\
(95 \% \mathrm{Cl})^{\mathrm{a}}\end{array}$ & $p$ value & $\begin{array}{l}\text { Prevalence } \\
(\%)\end{array}$ & $\begin{array}{l}\text { Adjusted OR } \\
(95 \% \mathrm{Cl})^{\mathrm{a}}\end{array}$ & $p$ value & $\begin{array}{l}\text { Prevalence } \\
(\%)\end{array}$ & $\begin{array}{l}\text { Adjusted OR } \\
(95 \% \mathrm{Cl})^{\mathrm{a}}\end{array}$ & $p$ value \\
\hline Africa & 67546 & & & & & & & & & \\
\hline$P_{50-} P_{74}$ & 15747 & 1.8 & $1 \cdot 00$ & - & $5 \cdot 5$ & $1 \cdot 00$ & - & $1 \cdot 9$ & $1 \cdot 00$ & - \\
\hline$P_{75}-P_{89}$ & 10150 & 2.3 & $1.31(1.08,1.59)$ & 0.0067 & $6 \cdot 5$ & $1 \cdot 23(1 \cdot 10,1 \cdot 38)$ & $0 \cdot 0002$ & $1 \cdot 8$ & $1 \cdot 18(0 \cdot 97,1 \cdot 44)$ & $0 \cdot 1032$ \\
\hline$P_{90}-P_{94}$ & 5247 & 2.5 & $1.36(1.08,1.72)$ & 0.0093 & $6 \cdot 0$ & $1 \cdot 25(1 \cdot 09,1 \cdot 44)$ & $0 \cdot 0019$ & $1 \cdot 8$ & $1 \cdot 39(1 \cdot 08,1 \cdot 78)$ & $0 \cdot 0106$ \\
\hline$P_{95}-P_{96}$ & 1820 & 3.6 & $1.91(1.40,2.60)$ & $<0 \cdot 0001$ & $6 \cdot 8$ & $1 \cdot 46(1 \cdot 18,1 \cdot 79)$ & $0 \cdot 0004$ & $1 \cdot 2$ & $1 \cdot 02(0 \cdot 64,1 \cdot 62)$ & 0.9335 \\
\hline$\geq P_{97}$ & 6851 & 3.8 & $2.00(1.64,2.44)$ & $<0 \cdot 0001$ & $9 \cdot 0$ & $2 \cdot 17(1 \cdot 92,2 \cdot 44)$ & $<0 \cdot 0001$ & $1 \cdot 7$ & $1 \cdot 86(1 \cdot 46,2 \cdot 37)$ & $<0 \cdot 0001$ \\
\hline Asia & 91595 & & & & & & & & & \\
\hline$P_{50}-P_{74}$ & 18066 & 12.3 & $1 \cdot 00$ & - & $12 \cdot 5$ & $1 \cdot 00$ & - & $2 \cdot 6$ & $1 \cdot 00$ & - \\
\hline$P_{75}-P_{89}$ & 14694 & 12.1 & $1.08(1.00,1.18)$ & 0.0611 & $12 \cdot 8$ & $1 \cdot 11(1 \cdot 03,1 \cdot 19)$ & 0.0056 & $2 \cdot 5$ & $1 \cdot 08(0 \cdot 93,1 \cdot 25)$ & $0 \cdot 3198$ \\
\hline$P_{90}-P_{94}$ & 5955 & 16.1 & $1.22(1.10,1.36)$ & 0.0002 & $14 \cdot 9$ & $1 \cdot 37(1 \cdot 25,1 \cdot 51)$ & $<0 \cdot 0001$ & $2 \cdot 2$ & $1 \cdot 05(0 \cdot 86,1 \cdot 29)$ & $0 \cdot 6286$ \\
\hline$P_{95}-P_{96}$ & 3030 & 14.5 & $1.31(1.14,1.51)$ & 0.0002 & $13 \cdot 4$ & $1 \cdot 60(1 \cdot 41,1 \cdot 82)$ & $<0 \cdot 0001$ & $3 \cdot 2$ & $1 \cdot 67(1 \cdot 32,2 \cdot 11)$ & $<0.0001$ \\
\hline$\geq P_{97}$ & 9574 & 18.1 & $1.65(1.50,1.81)$ & $<0 \cdot 0001$ & $17 \cdot 2$ & $1 \cdot 90(1 \cdot 75,2 \cdot 06)$ & $<0 \cdot 0001$ & $3 \cdot 0$ & $1 \cdot 55(1 \cdot 31,1 \cdot 83)$ & $<0 \cdot 0001$ \\
\hline Latin America & 87518 & & & & & & & & & \\
\hline$P_{50}-P_{74}$ & 20003 & 15.8 & $1 \cdot 00$ & - & $12 \cdot 3$ & $1 \cdot 00$ & - & $1 \cdot 3$ & $1 \cdot 00$ & - \\
\hline$P_{75}-P_{89}$ & 12912 & 17.7 & $1.11(1.03,1.19)$ & 0.0062 & $12 \cdot 7$ & $1 \cdot 08(1 \cdot 01,1 \cdot 17)$ & $0 \cdot 0291$ & $1 \cdot 3$ & $1 \cdot 13(0 \cdot 91,1 \cdot 39)$ & $0 \cdot 2628$ \\
\hline$P_{90}-P_{94}$ & 5227 & 19.9 & $1.27(1.16,1.40)$ & $<0 \cdot 0001$ & $13 \cdot 8$ & $1 \cdot 33(1 \cdot 21,1 \cdot 47)$ & $<0 \cdot 0001$ & $1 \cdot 7$ & $1 \cdot 55(1 \cdot 20,2 \cdot 02)$ & $0 \cdot 0010$ \\
\hline$P_{95}-P_{96}$ & 2242 & 21.7 & $1.42(1.24,1.63)$ & $<0 \cdot 0001$ & $15 \cdot 0$ & $1 \cdot 45(1 \cdot 26,1 \cdot 66)$ & $<0 \cdot 0001$ & $1 \cdot 2$ & $1 \cdot 34(0 \cdot 88,2 \cdot 06)$ & $0 \cdot 1770$ \\
\hline$\geq P_{97}$ & 6284 & 25.2 & $1.65(1.51,1.80)$ & $<0 \cdot 0001$ & $15 \cdot 5$ & $1 \cdot 84(1 \cdot 68,2 \cdot 02)$ & $<0 \cdot 0001$ & $1 \cdot 2$ & $1 \cdot 34(1 \cdot 01,1 \cdot 78)$ & 0.0402 \\
\hline
\end{tabular}

${ }^{a}$ All estimates were based on two-level logistic regression models. Facilities represent units at level two and individuals within facilities are observations at level one. We adjusted for country, maternal age, marital status, education (total years of school attendance), obesity, diabetes, parity, infant sex and gestational age

America, respectively. The rise in PMMI risks lagged behind that of MMMI in all three regions (Fig. 2). The aORs of PMMI reached $2 \cdot 0$ when birthweight was greater than $4200 \mathrm{~g}$ in Asia and $5000 \mathrm{~g}$ in Africa. In Latin America, birthweight of 4500-4999 g corresponded to the aOR of PMMI 1.78 (95\% CI: $1 \cdot 16,2 \cdot 74)$.
When birthweight was greater than $5000 \mathrm{~g}$, aOR rose dramatically to $7 \cdot 40$ (95\% CI: 3.5, 15 66) (Table 4).

When we excluded prelabor caesarean deliveries or restricted the analyses to vaginal deliveries, the results remained essentially unchanged (Additional file 3: Tables S3 and S4).
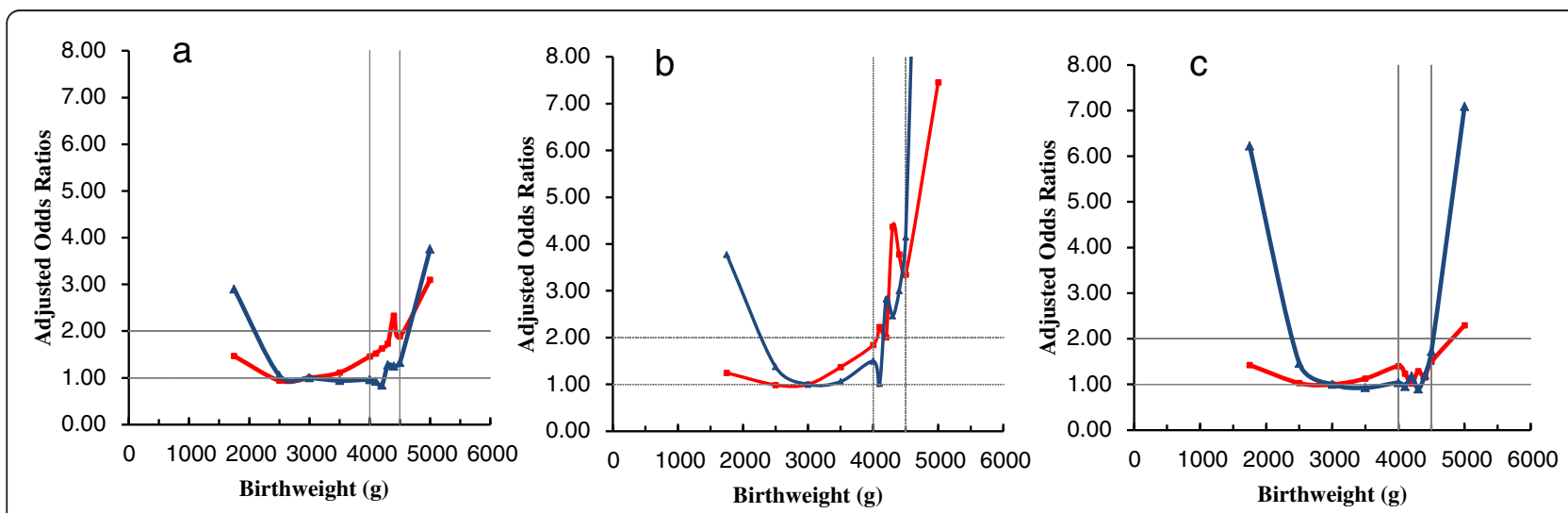

- Maternal mortality and morbidity index

Perinatal mortality and morbidity index

Fig. 2 Adjusted odds ratios of maternal and perinatal mortality and morbidity by birthweight in singleton term births. a: Africa b: Asia c: Latin America reference category: $3000-3499 \mathrm{~g}$ 
Table 4 Prevalence and odds ratios of maternal and perinatal mortality and morbidity by birthweight

\begin{tabular}{|c|c|c|c|c|c|c|c|}
\hline \multirow{2}{*}{$\begin{array}{l}\text { Birthweight } \\
\text { (g) }\end{array}$} & \multirow[t]{2}{*}{$\mathrm{N}$} & \multicolumn{3}{|c|}{ Maternal mortality and morbidity } & \multicolumn{3}{|c|}{ Perinatal mortality and morbidity } \\
\hline & & Prevalence (\%) & Adjusted OR $(95 \% \mathrm{Cl})^{\mathrm{a}}$ & $p$ value & Prevalence (\%) & Adjusted OR $(95 \% \mathrm{Cl})^{\mathrm{a}}$ & $p$ value \\
\hline Africa & 67546 & & $N=67546$ & & & & \\
\hline 3000-3499 & 28788 & $6 \cdot 0$ & $1 \cdot 00$ & - & $4 \cdot 6$ & $1 \cdot 00$ & - \\
\hline 3500-3999 & 15824 & $7 \cdot 2$ & $1 \cdot 10(1 \cdot 01,1 \cdot 20)$ & $0 \cdot 0289$ & $4 \cdot 2$ & $0 \cdot 94(0 \cdot 85,1 \cdot 05)$ & $0 \cdot 2718$ \\
\hline $4000-4099$ & 2154 & $9 \cdot 1$ & $1 \cdot 431 \cdot 20,1 \cdot 70)$ & $0 \cdot 0001$ & $4 \cdot 0$ & $0 \cdot 97(0 \cdot 76,1 \cdot 23)$ & $0 \cdot 7780$ \\
\hline $4100-4199$ & 670 & $10 \cdot 6$ & $1 \cdot 48(1 \cdot 12,1 \cdot 96)$ & $0 \cdot 0061$ & $3 \cdot 4$ & $0.93(0 \cdot 60,1 \cdot 44)$ & $0 \cdot 7424$ \\
\hline $4200-4299$ & 686 & $11 \cdot 7$ & $1 \cdot 59(1 \cdot 22,2 \cdot 07)$ & $0 \cdot 0006$ & $3 \cdot 4$ & $0 \cdot 84(0 \cdot 55,1 \cdot 31)$ & $0 \cdot 4473$ \\
\hline $4300-4399$ & 377 & $14 \cdot 3$ & $1 \cdot 68(1 \cdot 21,2 \cdot 34)$ & $0 \cdot 0021$ & $5 \cdot 0$ & $1 \cdot 29(0 \cdot 79,2 \cdot 09)$ & $0 \cdot 3122$ \\
\hline $4400-4499$ & 239 & $15 \cdot 5$ & $2 \cdot 26(1 \cdot 51,3 \cdot 39)$ & $0 \cdot 0001$ & $3 \cdot 8$ & $1 \cdot 26(0 \cdot 63,2 \cdot 49)$ & $0 \cdot 5147$ \\
\hline 4500-4999 & 648 & $15 \cdot 0$ & $1 \cdot 84(1 \cdot 44,2 \cdot 36)$ & $<0 \cdot 0001$ & $5 \cdot 4$ & $1 \cdot 34(0 \cdot 93,1 \cdot 93)$ & $0 \cdot 1216$ \\
\hline$\geq 5000$ & 146 & $21 \cdot 2$ & $3 \cdot 01(1 \cdot 91,4 \cdot 74)$ & $<0 \cdot 0001$ & $14 \cdot 4$ & $3 \cdot 79(2 \cdot 28,6 \cdot 30)$ & $<0 \cdot 0001$ \\
\hline Asia & 91595 & & $N=91595$ & & & & \\
\hline 3000-3499 & 36479 & $4 \cdot 7$ & $1 \cdot 00$ & - & $1 \cdot 7$ & $1 \cdot 00$ & - \\
\hline 3500-3999 & 13539 & $5 \cdot 4$ & $1 \cdot 38(1 \cdot 24,1 \cdot 52)$ & $<0 \cdot 0001$ & $1 \cdot 6$ & $1 \cdot 05(0 \cdot 90,1 \cdot 24)$ & 0.5196 \\
\hline 4000-4099 & 945 & $5 \cdot 4$ & $1 \cdot 86(1 \cdot 35,2 \cdot 56)$ & 0.0002 & $2 \cdot 1$ & $1 \cdot 49(0 \cdot 94,2 \cdot 35)$ & $0 \cdot 0891$ \\
\hline $4100-4199$ & 492 & $6 \cdot 7$ & $2 \cdot 25(1 \cdot 50,3 \cdot 38)$ & $0 \cdot 0001$ & $1 \cdot 2$ & $1 \cdot 01(0 \cdot 45,2 \cdot 29)$ & 0.9812 \\
\hline $4200-4299$ & 320 & $5 \cdot 0$ & $2 \cdot 03(1 \cdot 17,3 \cdot 52)$ & $0 \cdot 0122$ & $4 \cdot 1$ & $2 \cdot 80(1 \cdot 58,4 \cdot 99)$ & 0.0005 \\
\hline $4300-4399$ & 183 & $11 \cdot 5$ & $4 \cdot 42(2 \cdot 60,7 \cdot 50)$ & $<0 \cdot 0001$ & $3 \cdot 3$ & $2 \cdot 44(1 \cdot 06,5 \cdot 64)$ & $0 \cdot 0366$ \\
\hline $4400-4499$ & 101 & $7 \cdot 9$ & $3 \cdot 81(1 \cdot 68,8 \cdot 60)$ & 0.0013 & $4 \cdot 0$ & $2 \cdot 99(1 \cdot 07,8 \cdot 34)$ & 0.0367 \\
\hline 4500-4999 & 200 & $10 \cdot 0$ & $3 \cdot 38(1 \cdot 99,5 \cdot 74)$ & $<0 \cdot 0001$ & $6 \cdot 0$ & $4 \cdot 12(2 \cdot 24,7 \cdot 56)$ & $<0 \cdot 0001$ \\
\hline$\geq 5000$ & 52 & $17 \cdot 3$ & $7 \cdot 51(3 \cdot 25,17 \cdot 33)$ & $<0 \cdot 0001$ & $26 \cdot 9$ & $28 \cdot 44(14 \cdot 81,54 \cdot 61)$ & $<0 \cdot 0001$ \\
\hline Latin America & 87518 & & $N=87518$ & & & & \\
\hline 3000-3499 & 38930 & $2 \cdot 4$ & $1 \cdot 00$ & - & $2 \cdot 1$ & $1 \cdot 00$ & - \\
\hline 3500-3999 & 20875 & $2 \cdot 6$ & $1 \cdot 15(1 \cdot 03,1 \cdot 28)$ & 0.0157 & $2 \cdot 0$ & $0.94(0.83,1 \cdot 06)$ & $0 \cdot 3390$ \\
\hline 4000-4099 & 1675 & $3 \cdot 0$ & $1 \cdot 44(1 \cdot 08,1 \cdot 94)$ & $0 \cdot 0145$ & $2 \cdot 3$ & $1 \cdot 07(0 \cdot 77,1 \cdot 49)$ & 0.6852 \\
\hline 4100-4199 & 1020 & $2 \cdot 6$ & $1 \cdot 28(0 \cdot 86,1 \cdot 90)$ & $0 \cdot 2229$ & $2 \cdot 2$ & $0.98(0 \cdot 64,1 \cdot 52)$ & 0.9422 \\
\hline $4200-4299$ & 757 & $2 \cdot 5$ & $1 \cdot 05(0 \cdot 65,1 \cdot 70)$ & $0 \cdot 8450$ & $2 \cdot 8$ & $1 \cdot 22(0 \cdot 78,1 \cdot 91)$ & 0.3719 \\
\hline 4300-4399 & 466 & $3 \cdot 0$ & $1 \cdot 34(0 \cdot 77,2 \cdot 31)$ & $0 \cdot 3014$ & $2 \cdot 1$ & $0.94(0.5,1 \cdot 77)$ & $0 \cdot 8429$ \\
\hline $4400-4499$ & 281 & $2 \cdot 8$ & $1 \cdot 19(0 \cdot 58,2 \cdot 46)$ & $0 \cdot 6381$ & $2 \cdot 8$ & $1 \cdot 25(0 \cdot 61,2 \cdot 56)$ & 0.5378 \\
\hline 4500-4999 & 569 & $3 \cdot 5$ & $1 \cdot 54(0 \cdot 96,2 \cdot 45)$ & $0 \cdot 0708$ & $4 \cdot 0$ & $1 \cdot 78(1 \cdot 16,2 \cdot 74)$ & $0 \cdot 0089$ \\
\hline$\geq 5000$ & 55 & $5 \cdot 5$ & $2 \cdot 36(0 \cdot 70,8 \cdot 00)$ & $0 \cdot 1671$ & $16 \cdot 4$ & $7 \cdot 40(3 \cdot 50,15 \cdot 66)$ & $<0 \cdot 0001$ \\
\hline
\end{tabular}

${ }^{a}$ All estimates were based on two-level logistic regression models. Facilities represent units at level two and individuals within facilities are observations at level one. We adjusted for country, maternal age, marital status, education (total years of school attendance), obesity, diabetes, parity, infant sex, and gestational age

Table 5 shows that the risks of MMMI and PMMI in infants with a birthweight greater than the $95^{\text {th }}$ percentile increased slightly compared with that of birthweight at the $50^{\text {th }}-75^{\text {th }}$ percentiles in all three regions. When birthweight was at the $97^{\text {th }}$ percentile or higher, the aORs of MMMI and PMMI increased significantly, but none exceeded 2.0 in any region. In addition to the equation-derived global reference birthweight percentiles, we also used the empirical country- and gestationalage-specific birthweight percentile of the study population as cut-off points. The results were essentially unchanged (not shown).
We conducted sensitivity analyses using mortality and morbidity as two separate outcomes. The mortality included maternal and perinatal deaths. For morbidity, the occurrence of any components of MMMI and PMMI (excluding maternal or perinatal death) was considered as a positive event. As maternal and perinatal mortality were rare, no significant differences in mortality were found in the subgroups of infants with a birthweight greater than $4500 \mathrm{~g}$, or the $97^{\text {th }}$ birthweight percentile compared with the reference groups. However, results were similar for maternal and perinatal morbidity after excluding maternal or perinatal death (data no shown). 
Table 5 Prevalence and odds ratios of maternal and perinatal mortality and morbidity by birthweight percentile

\begin{tabular}{|c|c|c|c|c|c|c|c|}
\hline \multirow{2}{*}{$\begin{array}{l}\text { Birthweight } \\
\text { percentile }\end{array}$} & \multirow[t]{2}{*}{$N$} & \multicolumn{3}{|c|}{ Maternal mortality and morbidity } & \multicolumn{3}{|c|}{ Perinatal mortality and morbidity } \\
\hline & & Prevalence (\%) & Adjusted OR $(95 \% \mathrm{Cl})^{\mathrm{a}}$ & $p$ value & Prevalence (\%) & Adjusted OR $(95 \% \mathrm{Cl})^{\mathrm{a}}$ & $p$ value \\
\hline Africa & 67546 & & & & & & \\
\hline$P_{50}-P_{74}$ & 15747 & $6 \cdot 3$ & $1 \cdot 00$ & - & $4 \cdot 3$ & $1 \cdot 00$ & - \\
\hline$P_{75}-P_{89}$ & 10150 & $6 \cdot 6$ & $1 \cdot 17(1 \cdot 05,1 \cdot 31)$ & 0.0056 & $4 \cdot 3$ & $0 \cdot 98(0 \cdot 86,1 \cdot 11)$ & $0 \cdot 7055$ \\
\hline$P_{90}-P_{94}$ & 5247 & $5 \cdot 7$ & $1 \cdot 07(0 \cdot 93,1 \cdot 24)$ & $0 \cdot 3383$ & $5 \cdot 0$ & $1 \cdot 07(0 \cdot 92,1 \cdot 25)$ & $0 \cdot 3900$ \\
\hline$P_{95}-P_{96}$ & 1820 & $6 \cdot 6$ & $1 \cdot 31(1 \cdot 05,1 \cdot 62)$ & 0.0144 & $5 \cdot 4$ & $1 \cdot 15(0 \cdot 91,1 \cdot 45)$ & $0 \cdot 2301$ \\
\hline$\geq P_{97}$ & 6851 & $7 \cdot 2$ & $1 \cdot 54(1 \cdot 36,1 \cdot 75)$ & $<0 \cdot 0001$ & $5 \cdot 8$ & $1 \cdot 10(0 \cdot 96,1 \cdot 26)$ & $0 \cdot 1794$ \\
\hline Asia & 91595 & & & & & & \\
\hline$P_{50}-P_{74}$ & 18066 & $5 \cdot 0$ & $1 \cdot 00$ & - & $1 \cdot 8$ & $1 \cdot 00$ & - \\
\hline$P_{75}-P_{89}$ & 14694 & $5 \cdot 1$ & $1 \cdot 03(0 \cdot 92,1 \cdot 15)$ & 0.5834 & $2 \cdot 0$ & $1 \cdot 02(0 \cdot 86,1 \cdot 19)$ & 0.8529 \\
\hline$P_{90}-P_{94}$ & 5955 & $4 \cdot 8$ & $1 \cdot 04(0 \cdot 89,1 \cdot 21)$ & 0.6389 & $1 \cdot 7$ & $0 \cdot 88(0 \cdot 70,1 \cdot 11)$ & $0 \cdot 2784$ \\
\hline$P_{95}-P_{96}$ & 3030 & $5 \cdot 1$ & $1 \cdot 20(0 \cdot 98,1 \cdot 46)$ & 0.0742 & $2 \cdot 4$ & $1 \cdot 08(0 \cdot 83,1 \cdot 41)$ & 0.5513 \\
\hline$\geq P_{97}$ & 9574 & $6 \cdot 4$ & $1 \cdot 41(1 \cdot 25,1 \cdot 59)$ & $<0.0001$ & $2 \cdot 5$ & $1 \cdot 12(0 \cdot 94,1 \cdot 34)$ & $0 \cdot 1909$ \\
\hline Latin America & 87518 & & & & & & \\
\hline$P_{50}-P_{74}$ & 20003 & $2 \cdot 4$ & $1 \cdot 00$ & - & $2 \cdot 0$ & $1 \cdot 00$ & - \\
\hline$P_{75}-P_{89}$ & 12912 & $2 \cdot 7$ & $1 \cdot 13(0 \cdot 98,1 \cdot 30)$ & $0 \cdot 1014$ & $2 \cdot 2$ & $1 \cdot 10(0 \cdot 94,1 \cdot 29)$ & $0 \cdot 2236$ \\
\hline$P_{90}-P_{94}$ & 5227 & $2 \cdot 6$ & $1 \cdot 06(0 \cdot 87,1 \cdot 29)$ & 0.5709 & $1 \cdot 8$ & $0 \cdot 89(0 \cdot 71,1 \cdot 12)$ & 0.3257 \\
\hline$P_{95}-P_{96}$ & 2242 & $2 \cdot 9$ & $1 \cdot 21(0 \cdot 92,1 \cdot 58)$ & $0 \cdot 1744$ & $2 \cdot 1$ & $0 \cdot 98(0 \cdot 72,1 \cdot 34)$ & 0.9162 \\
\hline$\geq P_{97}$ & 6284 & $3 \cdot 0$ & $1 \cdot 23(1 \cdot 03,1 \cdot 47)$ & $0 \cdot 0215$ & $2 \cdot 8$ & $1 \cdot 31(1 \cdot 09,1 \cdot 58)$ & $0 \cdot 0042$ \\
\hline
\end{tabular}

${ }^{a}$ All estimates based on two-level logistic regression models. Facilities represent units at level two and individuals within facilities are observations at level one. We adjusted for country, maternal age, marital status, education (total years of school attendance), obesity, diabetes, parity, infant sex, and gestational age

Similar trends for the risks of MMMI and PMMI by birthweight and birthweight percentile were found when we restricted the analyses to countries with less than $10 \%$ of missing value for BMI (Additional file 3: Tables S5 and S6). When maternal obesity and diabetes were excluded from the regression models, aORs became larger for most of the subgroups, but the patterns remained the same in the three regions (Additional file 3: Tables S7 and S8).

\section{Discussion}

Our results indicate that there is a significant increase in adverse maternal and perinatal outcomes when the birthweight of term infants (37-42 weeks) reaches $4500 \mathrm{~g}$ in African and Latin America, and $4000 \mathrm{~g}$ in Asia. These cut-offs could, therefore, be used to define 'macrosomia' in these settings. Our findings do not support using LGA as a new definition for macrosomia because LGA was less predictive of adverse outcomes.

The use of the cut-off point of $4500 \mathrm{~g}$ to define macrosomia is consistent with results of previous studies of Caucasians populations [1,9], and supports the definition of the American Congress of Obstetricians and Gynecologists [1]. Based on analyses of national datasets of the United States, Zhang et al. [7], found that infants with birthweight of 4500-4999 g were at significantly increased risks of stillbirth, neonatal mortality (especially because of birth asphyxia), morbidity, and caesarean delivery as a consequence of either slow labor progress or non-reassuring cardiotocography. Ye et al. [9] used the same database also found that risks of MMMI or PMMI did not increased significantly until birthweight was at the $97^{\text {th }}$ percentile or higher. A birthweight cut-off points irrespective of gestational age (4500 g in Whites, $4300 \mathrm{~g}$ in Blacks and Hispanics) is more predictive of mortality and morbidity outcomes than the $97^{\text {th }}$ percentile for a given gestational age.

We used an OR of 2.0 for either MMMI or PMMI as a priori criterion to identify clinically important macrosomia. Though arbitrary, this cut-off point was also used in a study by Boulet et al. [18] in defining clinically important fetal growth restriction. In the randomized trial of the Twin Birth Study Collaborative Group, a relative risk of 0.5 was also used to justify the smallest clinically important difference between the planned caesarean delivery group and control group [19], equivalent to two-fold increase (or decrease) of risks.

Using the concept of birthweight percentile at a given gestational age (i.e., LGA) as the definition of macrosomia has been proposed in recent years $[2,10,21]$. However, our study shows that it has a poor prediction of adverse maternal and perinatal outcomes. This may be partially attributable to the imprecise estimation of gestational age, which has resulted in misclassifications of 
macrosomia. Thus, a definition based on birthweight would be more practical, especially in settings where accurate estimation of gestational age may be difficult.

We observed that risks of elective and intrapartum caesarean section increased significantly for infants with a birthweight greater than $4000 \mathrm{~g}$ in the selected facilities of all three regions. However, the risks of or vacuum extraction did not increase prominently, which suggested that obstetricians may have a tendency for operative delivery when faced with a suspiciously large fetus and slow labor progress. Therefore, an estimated birthweight of $4000 \mathrm{~g}$ may be a useful indicator for difficult labour. We also found that risks of MMMI and PMMI did not change substantially after excluding elective caesarean deliveries, suggesting that suspected macrosomic cases may not benefits from elective caesarean section in a meaningful way. This was also demonstrated in other studies [22, 23].

We also observed that risks of MMMI increased prior to that of PMMI in three regions. This was consistent with the three-level definition of macrosomia proposed by Boulet et al. [3]: Grade 1 ( $>4000 \mathrm{~g}$ ) to identify increased risks of labour and newborn complications; Grade $2(>4500 \mathrm{~g})$ to predict neonatal morbidity; and Grade $3(>5000 \mathrm{~g})$ to predict infant mortality. The major causes of maternal mortality and morbidity for mothers of macrosomic babies included uterine atony, prolonged labour, haemorrhage, vesico-vaginal/recto-vaginal fistula and severe perineal laceration [21]. These complications contributed to MMMI in our study. The most frequently severe adverse outcome was admission to an intensivecare unit $(5.0 \%)$, followed by blood transfusion $(2.0 \%)$ and third or fourth degree perineal laceration $(1.5 \%)$ for suspected macrosomia infants.

Macrosomia represents a significant obstetric challenge. The definition of macrosomia has important clinical, medicolegal and cost implications. Therefore, it should be evidence-based, particularly in resources limited countries where skilled birth attendants and caesarean delivery may not be readily available, and hospital transfer and special care are costly. To the best of our knowledge, this is the first study to search for an evidence-based definition of macrosomia in LMICs.

However, our study has several limitations. First, the significance of the definition of macrosomia using birthweight cut-off point in obstetric management is limited by the inaccuracy in birthweight estimation. Either ultrasound or clinical prediction of birthweight is not accurate enough to serve as the basis for obstetric decision making. But obstetricians are prone to operative delivery when faced with a suspected large fetus, which may lead to unnecessary cesarean [24].

Second, despite the standardization of data collection, participating facilities may have different labour management protocols. Inter-institutional variability is inevitable due to the nature of a multinational study. For example, screening for diabetes in pregnancy is not available in all facilities, particularly in Africa [2], and therefore underestimation of gestational diabetes is likely. However, our sensitivity analysis, which excluded the variable "diabetes" from the regression models, showed a similar result to that of the fully adjusted models.

Third, information on maternal height and weight was problematic in two aspects. More than $10 \%$ of data for height and/or weight were missing for some countries. In addition, maternal weight was defined differently across regions: in Africa and Latin America it was referred to as the first recorded weight at the first antenatal care visit while in Asia it was defined as the last recorded weight before delivery. In theory, the last recorded maternal weight would better control for the potential confounding effects of gestational weight gain in Asia [25]. However, the results of the sensitivity analysis, which excluded those countries or the variable "obesity" from the regression model indicated that the limitation did not affect the conclusion.

Finally, the sample used in the survey was selected from facilities with more than 1000 deliveries per year and where caesarean sections were available [11]. Selection bias is possible, especially in certain LMICs where an institutional delivery rate is low. Therefore, the generalizability of our findings may be limited.

\section{Conclusions}

A population-specific definition of macrosomia using birthweight cut-off points (4500 g in Africa and Latin America, $4000 \mathrm{~g}$ in Asia) for term infants at 37-42 gestational weeks is more closely associated with maternal and perinatal mortality and morbidity. This definition is also easier to apply than that based on birthweight percentile for a given gestational age. The use of an evidence-based definition of macrosomia may improve obstetric and perinatal care, especially in resourcelimited settings in LMICs.

\section{Additional files}

Additional file 1: Table S1. Maternal characteristics by region and birthweight in singleton term births. (PDF 365 kb)

Additional file 2: Table S2. Indications for caesarean section in suspected macrosomic infants. (PDF 187 kb)

Additional file 3: Tables S3-S8. Results of sensitivity analyses. (PDF $577 \mathrm{~kb}$ )

\section{Abbreviations}

aORs: adjusted odds ratios; BMl: body mass index; ICU: intensive-care unit; LGA: large-for-gestational-age; LMICs: low- and middle-income countries; MMMI: maternal mortality and morbidity index; ORs: odds ratios; PMMI: perinatal mortality and morbidity index. 


\section{Competing interests}

The authors declare that they have no competing interests.

\section{Authors' contributions}

JFY and JZ participated in the study design. JFY analyzed the data and drafted the manuscript. JZ co-wrote the paper. MRT, EO, KJ, CP, EOP, PL, NM, ML, RM, ÖT, FF, HY, JPS, JPV provided critical comments and valuable suggestions on the manuscript. All authors contributed to the discussion, read and approved the final version of the manuscript.

\section{Acknowledgements}

WHO Global Survey on Maternal and Perinatal Health (WHOGS, 2004-08) was financially supported by the UNDP/UNFPA/WHO/World Bank Special Programme of Research, Development, and Research Training in Human Reproduction (HRP); WHO; United States Agency for International Development (USAID); Ministry of Health, Labour and Welfare of Japan; Ministry of Public Health of the People's Republic of China; and the Indian Council of Medical Research, India. The authors of this secondary analysis are grateful to all those who contributed to the project design and implementation, including researchers, study coordinators, staff from the $\mathrm{WHO}$ offices and other partners of the study. The named authors alone are responsible for the views expressed in this manuscript, which does not necessarily represent the decisions or the stated policy of the WHO. The authors would like to thank Emma Barber-an English-language editor based at the National Center for Child Health and Development, Tokyo, Japan—for her editorial support.

This work was supported by National Natural Science Foundation of China (No. 31101179). The funding agency had no role in the: design and conduct of the study; collection, management, analysis, and interpretation of the data; and preparation, review, or approval of the manuscript; and decision to submit the manuscript for publication.

\section{Author details}

${ }^{1}$ Ministry of Education-Shanghai Key Laboratory of Children's Environmental Health, Xinhua Hospital, Shanghai Jiao Tong University School of Medicine, Shanghai, China. ${ }^{2}$ UNDP • UNFPA • UNICEF • WHO • World Bank Special Programme of Research, Development and Research Training in Human Reproduction, Department of Reproductive Health and Research, World Health Organization, Geneva, Switzerland. ${ }^{3}$ Department of Internal Medicine, Post Graduate program of Evidence Based Healthcare, São Paulo Federal University, São Paulo, SP, Brazil. ${ }^{4}$ Department of Health Policy, National Center for Child Health and Development, Tokyo, Japan. ${ }^{5}$ Family Health Bureau, Ministry of Health, 231, De Saram Place, Colombo 10, Sri Lanka. ${ }^{6}$ Department of Pediatrics, Ribeirão Preto Medical School, University of São Paulo, Ribeirão Preto, SP, Brazil. ${ }^{7}$ Center for Population Health Research, National Institute of Public Health, Cuernavaca, Mexico. ${ }^{8}$ Department of Obstetrics \& Gynaecology, Faculty of Medicine, Khon Kaen University, Khon Kaen, Thailand. 'Division of Lifecourse Epidemiology, Department of Social Medicine, National Center for Child Health and Development, Tokyo, Japan. ${ }^{10}$ Department of Biostatistics \& Demography, Faculty of Public Health, Khon Kaen University, Khon Kaen, Thailand. ${ }^{11} \mathrm{~S} c h o o l$ of Public Health, Guilin Medical College, Guangxi, China. ${ }^{12}$ Department of Social Medicine, Ribeirão Preto Medical School, University of São Paulo, Ribeirão Preto, SP, Brazil.

Received: 1 May 2015 Accepted: 25 November 2015

\section{Published online: 03 December 2015}

\section{References}

1. Chatfield J. ACOG issues guidelines on fetal macrosomia. American College of Obstetricians and Gynecologists. Am Fam Physician. 2001;64(1):169-70.

2. Koyanagi A, Zhang J, Dagvadorj A, Hirayama F, Shibuya K, Souza JP, et al. Macrosomia in 23 developing countries: an analysis of a multicountry, facility-based, cross-sectional survey. Lancet. 2013;381(9865):476-83.

3. Boulet SL, Alexander GR, Salihu HM, Pass M. Macrosomic births in the United States: Determinants, outcomes, and proposed grades of risk. Am J Obstet Gynecol. 2003;188(5):1372-8.

4. Dennedy MC, Dunne F. Macrosomia: defining the problem worldwide. Lancet. 2013;381(9865):435-6.

5. Martorell R, Khan LK, Hughes ML, Grummer-Strawn LM. Obesity in women from developing countries. Eur J Clin Nutr. 2000;54(3):247-52.
6. Roglic G. Diabetes in women: the global perspective. Int J Gynaecol Obstet. 2009;104 Suppl 1:S11-13.

7. Zhang X, Decker A, Platt RW, Kramer MS. How big is too big? The perinatal consequences of fetal macrosomia. Am J Obstet Gynecol. 2008;198(5):517. e511-516.

8. Stotland NE, Caughey AB, Breed EM, Escobar GJ. Risk factors and obstetric complications associated with macrosomia. Int J Gynaecol Obstet. 2004;87(3):220-6.

9. Ye J, Zhang L, Chen Y, Fang F, Luo Z, Zhang J. Searching for the definition of macrosomia through an outcome-based approach. PLoS One. 2014;9(6), e100192.

10. Pasupathy D, McCowan LM, Poston L, Kenny LC, Dekker GA, North RA, et al. Perinatal outcomes in large infants using customised birthweight centiles and conventional measures of high birthweight. Paediatr Perinat Epidemiol. 2012;26(6):543-52.

11. Shah A, Faundes A, Machoki Ml, Bagaglia V, Amokrane F, Donner A, et al. Methodological considerations in implementing the WHO Global Survey for Monitoring Maternal and Perinatal Health. Bull World Health Organ. 2008; 86(2):126-31.

12. Souza JP, Cecatti JG, Faundes A, Morais SS, Villar J, Carroli G, et al. Maternal near miss and maternal death in the World Health Organization's 2005 global survey on maternal and perinatal health. Bulletin of the World Health Organization. 2010;88(2):113-119.

13. Vangen S, Stoltenberg C, Skjaerven R, Magnus P, Harris JR, Stray-Pedersen B. The heavier the better? Birthweight and perinatal mortality in different ethnic groups. Int J Epidemiol. 2002;31(3):654-60.

14. Wilcox A, Skjaerven R, Buekens P, Kiely J. Birth weight and perinatal mortality. A comparison of the United States and Norway. JAMA. 1995;273(9):709-11.

15. Wilcox AJ, Russell IT. Birthweight and perinatal mortality: II. On weightspecific mortality. Int J Epidemiol. 1983;12(3):319-25.

16. Wilcox AJ, Russell IT. Birthweight and perinatal mortality: III. Towards a new method of analysis. Int J Epidemiol. 1986;15(2):188-96.

17. Graafmans WC, Richardus JH, Borsboom GJ, Bakketeig L, Langhoff-Roos J, Bergsjo $P$, et al. Birth weight and perinatal mortality: a comparison of "optimal" birth weight in seven Western European countries. Epidemiology. 2002;13(5):569-74

18. Boulet SL, Alexander GR, Salihu HM, Kirby RS, Carlo WA. Fetal growth risk curves: defining levels of fetal growth restriction by neonatal death risk. Am J Obstet Gynecol. 2006;195(6):1571-7.

19. Barrett JF, Willan AR, Joseph KS. Planned cesarean or vaginal delivery for twin pregnancy. N Engl J Med. 2014;370(3):280.

20. Mikolajczyk RT, Zhang J, Betran AP, Souza JP, Mori R, Gulmezoglu AM, et al. A global reference for fetal-weight and birthweight percentiles. Lancet. 2011;377(9780):1855-61.

21. Henriksen T. The macrosomic fetus: a challenge in current obstetrics. Acta Obstet Gynecol Scand. 2008;87(2):134-45.

22. Rouse DJ, Owen J, Goldenberg RL, Cliver SP. The effectiveness and costs of elective cesarean delivery for fetal macrosomia diagnosed by ultrasound. JAMA. 1996;276(18):1480-6.

23. Sanchez-Ramos L, Bernstein S, Kaunitz AM. Expectant management versus labor induction for suspected fetal macrosomia: a systematic review. Obstet Gynecol. 2002;100(5 Pt 1):997-1002.

24. Chauhan SP, Grobman WA, Gherman RA, Chauhan VB, Chang G, Magann $E F$, et al. Suspicion and treatment of the macrosomic fetus: a review. Am J Obstet Gynecol. 2005;193(2):332-46.

25. Diouf I, Botton J, Charles MA, Morel O, Forhan A, Kaminski M, et al. Specific role of maternal weight change in the first trimester of pregnancy on birth size. Matern Child Nutr. 2014;10(3):315-26. 